\title{
Acquired Chylothorax
}

National Cancer Institute

\section{Source}

National Cancer Institute. Acquired Chylothorax. NCI Thesaurus. Code C98804.

Chylothorax that results from malignancies (usually lymphoma), trauma to the thoracic duct, tuberculosis, or sarcoidosis. 